INPLASY

PROTOCOL

To cite: Lai et al. Health

Knowledge among Type 2

Diabetes Patients in Malaysia:

A Systematic Review. Inplasy

protocol 202190044. doi:

10.37766/inplasy2021.9.0044

Received: 15 September 2021

Published: 15 September 2021

Corresponding author:

Pei Kuan Lai

laipeikuan@imu.edu.my

Author Affiliation:

International Medical

University.

Support: IMU Internal Grant.

Review Stage at time of this submission: The review has not yet started.

Conflicts of interest:

None declared.

\section{Health Knowledge among Type 2 Diabetes Patients in Malaysia: A Systematic Review}

\author{
Lai, PK'1 Teng, CL2; Mustapha, $\mathrm{FI}^{3}$.
}

Review question / Objective: To document the level of diabetes knowledge using Michigan Diabetes Knowledge Test (MDKT) among type 2 diabetes mellitus patients in Malaysia. Rationale: Diabetes mellitus is a chronic health condition which requires essential self-care activities and management. In order to perform self-care well, it is essential for individuals with diabetes to have sufficient levels of diabetes knowledge. Diabetes knowledge is one of the crucial elements to look into as part of the overall assessment of diabetic patients for many years. Inevitably, country-based diabetes knowledge systematic reviews are crucial to identify the knowledge gap amongst the T2DM in different countries. Therefore, this systematic review aims to summarise and report on current published evidence on the level of diabetes knowledge measured by the Michigan Diabetes Knowledge Test (MDKT) among T2DM patients in Malaysia. The findings from the reviews could provide useful information for healthcare providers in each country so they can better evaluate the knowledge of diabetes patients about their disease and subsequently plan interventions accordingly to enhance patients' knowledge.

INPLASY registration number: This protocol was registered with the International Platform of Registered Systematic Review and Meta-Analysis Protocols (INPLASY) on 15 September 2021 and was last updated on 15 September 2021 (registration number INPLASY202190044).

\section{INTRODUCTION}

Review question / Objective: o document the level of diabetes knowledge using Michigan Diabetes Knowledge Test (MDKT) among type 2 diabetes mellitus patients in Malaysia.

Rationale: Diabetes mellitus is a chronic health condition which requires essential self-care activities and management. In 
order to perform self-care well, it is essential for individuals with diabetes to have sufficient levels of diabetes knowledge. Diabetes knowledge is one of the crucial elements to look into as part of the overall assessment of diabetic patients for many years. Inevitably, country-based diabetes knowledge systematic reviews are crucial to identify the knowledge gap amongst the T2DM in different countries. Therefore, this systematic review aims to summarise and report on current published evidence on the level of diabetes knowledge measured by the Michigan Diabetes Knowledge Test (MDKT) among T2DM patients in Malaysia. The findings from the reviews could provide useful information for healthcare providers in each country so they can better evaluate the knowledge of diabetes patients about their disease and subsequently plan interventions accordingly to enhance patients' knowledge.

Condition being studied: Diabetes mellitus (DM) is a crucial public health issue worth paying attention to due to its increasing prevalence globally. According to the National Health and Morbidity Survey (NHMS) 2019, 3.9 million (18.3\%) Malaysians aged 18 years and above were found to have diabetes, which was a spike from the $13.4 \%$ diabetes prevalence rate in 2015. In other words, nearly one in five Malaysian adults are diabetics. The highest prevalence was found in the state of Negeri Sembilan (33.2\%), while the lowest prevalence was reported in Sabah $(9.8 \%)$. Indians had the highest prevalence (31.4\%), followed by Malays (21.6\%), Chinese (15.1\%). Type 2 diabetes mellitus (T2DM) has become more prevalent worldwide and its global prevalence among adults particularly was found to have doubled to 8.5\% over more than three decades. In Malaysia, the prevalence of T2DM has increased from $15.2 \%$ to $17.5 \%$ over the last half decade. According to the National Diabetes Registry (NDR) 2013-2019, there were 1,614,363 patients registered in the NDR, of which $99.3 \%$ were diagnosed with T2DM. The mean age of T2DM patients in the NDR was 63 years old, and the mean age at diagnosis for T2DM patients was 53 years old. The majority of patients were female $(57.1 \%)$ and Malay $(59.2 \%)$.

\section{METHODS}

Search strategy: We plan to search bibliographic databases for Malaysian diabetes studies that evaluated diabetes knowledge using the Michigan Diabetes Knowledge Test (MDKT). Bibliographic search will be performed using keywords "diabetes AND knowledge AND Malaysia". Studies using MDKT will be filtered from the retrieved eligible references using citation manager to ensure that only Malaysian studies done with MDKT are included for the final analysis. There is no limit to the year of publication for included studies.

Participant or population: Adult patients with type 2 diabetes mellitus.

Intervention: Michigan Diabetes Knowledge Test (MDKT).

Comparator: - Subgroups (e.g., age, gender, ethnicity, educational level, household incomes, etc). - Change in knowledge score by decade (year of publication). - Correlation between level of knowledge and HbA1c.

Study designs to be included: Crosssectional studies.

Eligibility criteria: - Studies conducted in Malaysia- Study participants are adult patients with type 2 diabetes mellitusStudies using Michigan Diabetes Knowledge Test (MDKT)- Cross-sectional studies.

Information sources: An extensive literature search will be carried out on electronic databases such as PubMed, ScienceDirect, CINAHL, and Google Scholar databases.

Main outcome(s): Knowledge level as assessed using Michigan Diabetes Knowledge Test (MDKT).

Additional outcome(s): Correlation with HbA1c. 
Data management: References retrieved will be managed using citation manager Endnote X8. The corresponding authors of eligible studies will be contacted to request for statistical datasets on MDKT.

Quality assessment / Risk of bias analysis: Two reviewers will independently screen titles and abstracts for eligible studies, followed by full-text reading for methodological validity. Ineligible studies will be excluded from full-text review, and the reasons for exclusion will be documented. Data will be extracted independently by two reviewers into a data extraction form. If multiple publications of the same study are retrieved, only the most recent relevant data will be included from these publications. The quality assessment of the published studies will be assessed using Joanna Briggs Institute (JBI) Critical Appraisal tools. The extraction form will be created following the Cochrane Handbook for Systematic Reviews of Interventions guidelines, with consensus from all the reviewers. A predesigned data extraction form will be used to collect details as follows: (1) authors with year published, (2) sample size, (3) participant characteristics, (4) settings, and (5) key findings.

Strategy of data synthesis: For quantitative data, where possible, meta-analysis will be performed using MedCalc Statistical Software. For meta-analysis of studies with a continuous measure (comparison of means between different sociodemographic groups), MedCalc uses the Hedges $g$ statistic as a formulation for the standardized mean difference under the fixed effects model or random effects model (the latter will be selected if $12>50 \%$ ).

Subgroup analysis: - Sociodemographic variables e.g. age, gender, ethnicity, educational level, household incomes, etc. - Change in knowledge score by decade (year of publication). - Correlation between level of knowledge and HbA1c.

Sensitivity analysis: None.

Language: English language.
Country(ies) involved: Malaysia.

Other relevant information: This systematic review will be prepared following PRISMAIPD and MOOSE guidelines.

Keywords: Type 2 diabetes mellitus, knowledge, Michigan Diabetes Knowledge Test (MDKT), Malaysia.

Contributions of each author:

Author 1 - Pei Kuan Lai - Protocol preparation; literature search and management; statistical analysis; manuscript preparation.

Email: laipeikuan@imu.edu.my

Author 2 - Cheong Lieng Teng - Protocol preparation; literature search and management; statistical analysis; manuscript preparation.

Email: cheonglieng_teng@imu.edu.my Author 3 - Feisul Idzwan Mustapha Protocol preparation; manuscript preparation.

Email: feisulidzwan@gmail.com 